\title{
Quais tipos de locais de trabalho participam de estudos para perda de peso?
}

\author{
What types of worksites participate in weight loss trials?
}

\section{Cuáles tipos de locales de trabajo participan de estudios para pérdida de peso?}

Recebido: 24/06/2013

Aprovado: 13/02/2014

\author{
Fabio Araújo Almeida ${ }^{1}$ \\ Sarah Stacy Wall ${ }^{2}$ \\ Russell Eugene Glasgow ${ }^{3}$ \\ Laura Ann Linnan ${ }^{4}$ \\ Brenda Marie Davy 5 \\ Jennie Linn Hill 6 \\ Wendy You ${ }^{7}$ \\ Paul Andrew Estabrooks ${ }^{8}$
}

O objetivo deste artigo é descrever o processo e os resultados associados com a adoção no estudo Worksite Weight Control (Controle de Peso no Local de Trabalho). Este é um estudo quantitativo desenvolvido no Estado da Virginia nos Estados Unidos entre os anos de 2007 e 2010. Um total de 119 locais de trabalho foram identificados como potencialmente elegíveis com base em tamanho, no acesso à internet e vontade de realizar um questionário breve de saúde no local de trabalho. Setenta e três foram elegíveis, 28 (38,3\%) foram inscritos, e 26 completaram os 12 meses de intervenção (35,6\%). Estes locais incluíram quatro instalações médicas $(14,3 \%)$, seis centros de distribuição e produção $(21,4 \%)$, cinco grupos profissionais das áreas de direito, publicidade, engenharia, vendas e suporte de tecnologia da informação $(17,9 \%)$, dois centros de atendimento $(7,1 \%)$, quatro faculdades e universidades pequenas $(14,3 \%)$, e sete agências governamentais $(25,0 \%)$. Não houve diferenças estatisticamente significativas nas taxas de adoção com base no tipo de local de trabalho. No entanto, houve tendências sugerindo que grupos profissionais e faculdades pequenas eram mais propensos a recusar a participação, sendo que as fábricas e agências governamentais eram mais aptos à participação. Os resultados sugerem que os programas foram aceitáveis para uma variedade

\footnotetext{
1 Assistente Social. Especialista em Práticas Comunitárias. Mestre em Serviço Social com ênfase em Prática Comunitária e Desenvolvimento Internacional. Doutor em Serviço Social. Professor dos Cursos de Mestrado e Doutorado em Nutrição Humana, Alimentação e Exercício. Universidade Virginia Tech., EUA. falmeida@vt.edu

2 Bacharel em Matemática. Mestre em Educação em Saúde. Pesquisadora do Departamento de Nutrição Humana, Alimentação e Exercício. Universidade Virginia Tech., EUA.

${ }_{3}^{3}$ Psicólogo. Mestre em Psicologia Clínica. Doutor em Psicologia Clínica. Professor do Programa de Residência em Cuidados de Atenção Primária e do Doutorado em Medicina. Universidade do Colorado, EUA.

4 Educadora em Saúde. Especialista em Educação em Saúde. Mestre em Educação em Saúde Pública. Doutora em Saúde e Comportamento Social. Professora do Mestrado em Saúde Pública e Doutorado em Saúde Comportamental e Educação em Saúde. Departamento de Saúde Comportamental e Educação em Saúde da Universidade da Carolina do Norte, EUA.

5 Nutricionista. Mestre em Fisiologia do Exercício. Doutora em Nutrição Humana. Professora dos Cursos de Mestrado e Doutorado em Nutrição Humana, Alimentação e Exercício. Universidade Virginia Tech., EUA.

${ }_{6}^{6}$ Psicóloga. Mestre em Cinesiologia. Doutora em Ciências da Saúde e Comportamentais. Professor dos Cursos de Mestrado e Doutorado em Nutrição Humana, Alimentação e Exercício. Universidade Virginia Tech., EUA.

7 Bacharel em Artes. Especialista em Comércio Internacional. Doutora em Economia Agrária. Departamento de Agricultura Aplicada. Professora do Mestrado em Agricultura e Economia Aplicada e do Doutorado em Economia. Universidade Virginia Tech., EUA.

8 Educador Físico. Mestre em Cinesiologia. Doutor em Cinesiologia. Professor dos Cursos de Mestrado e Doutorado em Nutrição Humana, Alimentação e Exercício. Universidade Virginia Tech., EUA.
} 
de tipos de local de trabalho, mas programas aplicados pela internet podem não ser tão atraentes para grupos profissionais e faculdades de pequeno porte.

Descritores: Promoção da saúde; Programas de redução de peso; Obesidade; Local de trabalho; Internet.

The goal of this paper is to describe the process and outcomes associated with adoption for the Worksite Weight Control Trial. This prospective study took place in Virginia, USA between the years 2007 and 2010. A total of 119 worksites were identified as potentially eligible, based on size, access to internet, and willingness to conduct a worksite wide brief health survey. Seventy-three were eligible, 28 (38.3\%) enrolled, and 26 completed the 12 months of intervention (35.6\%). These sites included four medical facilities (14.3\%), six manufacturing and distribution centers (21.4\%), five professional groups in law, advertising, engineering, sales, and information technology support (17.9\%), two call centers (7.1\%), 4 small colleges and universities (14.3\%), and seven governmental agencies (25.0\%). There were no statistically significant differences in adoption rates based on type. However, there were trends suggesting professional groups and small colleges were more likely to decline participation, and manufacturing sites and governmental agencies more likely to join. While these findings suggest the programs were acceptable to a variety of worksite types, internetdelivered programs may not be as attractive for professional groups and small colleges.

Descriptors: Health promotion; Weight reduction programs; Obesity; Workplace; Internet.

El objetivo de este artículo es describir el proceso y los resultados asociados con la adopción en el estudio Worksite Weight Control (Control de Peso en el Local de Trabajo). Este es un estudio cuantitativo desarrollado en el Estado de Virginia en los Estados Unidos entre los años de 2007 y 2010 . Un total de 119 locales de trabajo fueron identificados como potencialmente elegibles con base al tamaño, en el acceso a internet y voluntad de realizar unos cuestionarios breves de salud en el local de trabajo. Setenta y tres fueron elegibles, 28 (38,3\%) fueron inscriptos, y 26 completaron los 12 meses de intervención (35,6\%). Estos locales incluyeron cuatro instalaciones médicas (14,3\%), seis centros de distribución y producción $(21,4 \%)$, cinco grupos profesionales de las clases de derecho, publicidad, ingeniería, ventas y soporte de tecnología de la información $(17,9 \%)$, dos centros de atendimiento $(7,1 \%)$, cuatro facultades y universidades pequeñas $(14,3 \%)$, y siete agencias gubernamentales $(25,0 \%)$. No hubo diferencias estadística-mente significativa en las tasas de adopción con base al tipo de local de trabajo. Sin embargo, hubo tendencias sugiriendo que grupos profesionales y facultades pequeñas eran más propensos a recusar la participación, siendo que las fábricas y agencias gubernamentales eran más aptos a la participación. Los resultados sugieren que los programas fueron aceptables para una variedad de tipos de local de trabajo, pero programas aplicados por la internet pueden no ser tan atrayentes para grupos profesionales y facultades de pequeño porte.

Descriptores: Promoción de la salud; Programas de reducción de peso; Obesidad; Local de trabajo; Internet. 


\section{INTRODUÇÃO}

$\mathrm{D}$ adas as consequências negativas do excesso de peso e obesidade, a prevalência atual dessas condições é alarmante $^{1,2}$. Para lidar com a crescente epidemia de obesidade, programas de promoção da saúde realizados em locais de trabalho têm sido recomendados devido ao seu alcance potencial e impacto do apoio social $^{3-5}$.

Dentro do contexto do local de trabalho, estratégias para prevenir e tratar da obesidade têm se concentrado em ações de educação em saúde executados individualmente ou em pequenos grupos e com enfoque em estratégias de aquisição de conhecimento destinados a ajudar indivíduos a melhorarem suas práticas de alimentação e atividade física.

Esses programas têm sido criticados porque geralmente atingem uma pequena percentagem de trabalhadores (e raramente aqueles que poderiam/precisariam se beneficiar), são de curta duração, e têm efeitos geralmente pequenos, que não são sustentados $^{6-8}$. Além disso, muitos não relatam o tamanho do local de trabalho ${ }^{9}$, obstante aqueles que têm relatado, geralmente são realizados em grandes locais de trabalho ${ }^{10,11}$ fazendo com que generalizações sobre o seu benefício para outros locais de trabalho seja limitada9

Ademais, estas intervenções raramente relatam importantes questões sobre o percentual de locais e/ou agentes de intervenção que foram excluídos, que participaram e quão representativos eles poderiam ter sido $^{4,12}$. Esta falta de informação acerca da validade externa, muitas vezes torna difícil para os responsáveis pela tomada de decisão avaliar os programas e ou políticas a serem adotadas e pode explicar a falta de translação de programas de promoção da saúde com base no local de trabalho para a prática contínua.

Para contribuir no preenchimento dessa lacuna, foi realizado este estudo sobre a dimensão Adoção do modelo RE-AIM ${ }^{13}$ de dois programas baseados no local de trabalho para promoção da saúde, como parte do projeto Tailored Worksite Weight Control - Programas sob Medida em Locais de Trabalho para o Controle de Peso (Projeto

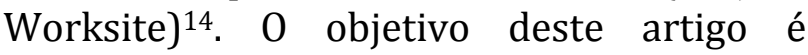
descrever o processo e os resultados associados com a adoção no estudo Worksite Weight Control (Controle de Peso no Local de Trabalho).

\section{MÉTODO}

Este artigo é parte de um ensaio randomizado controlado com dois grupos, com a intenção de investigar o alcance e a efetividade de dois programas de perda de peso no local de trabalho ${ }^{14}$.

O projeto Worksite testou a utilidade de uma intervenção individualmente orientada e baseada na Internet (programa INCENT), fundamentada na Teoria Social Ecológica, para reduzir o peso dos trabalhadores com excesso de peso e obesos, quando comparado com um programa menos intensivo de boletins trimestrais (programa Livin' My Weigh).

Os participantes do programa INCENT receberam suporte frequente por email, o que facilitava a fixação de metas, avaliações periódicas do peso corporal, além de incentivos monetários modestos com base na porcentagem do peso original perdido no final de cada trimestre durante os 12 meses do programa.

Os participantes do programa Livin' My Weigh (LMW) receberam quatro boletins informativos trimestrais com versões condensadas de materiais informativos utilizados no programa INCENT $\mathrm{e}$ foi oferecida a oportunidade de participar de quatro sessões de grupos de suporte destinados a complementar as informações incluídas nos boletins ao longo de um período de 12 meses. Para este artigo, apresentar-se-ão dados relacionados à identificação dos locais de trabalho, recrutamento e adesão.

Para ser elegível ao projeto, os locais de trabalho tiveram que: a) fornecer acesso à 
internet a todos os funcionários; b) ter entre 100 e 600 empregados; c) ter empregados fisicamente localizados em um único local com acesso centralizado para aferição de peso; d) concordar em realizar um questionário breve de saúde (Brief Health Survey - BHS) em todos funcionários.

0 recrutamento de locais de trabalho começou em agosto de 2007 e continuou até maio de 2010. Locais de trabalho potenciais foram identificados através de uma variedade de métodos, incluindo: 1) entrar em contato com câmaras de comércio locais e associações empresariais; 2) propaganda nos principais jornais na área de cobertura; 3) notícias pela televisão; 4) contatar operadoras de seguro de saúde; 5) usar buscas na internet em sites voltados para o desenvolvimento econômico nos municípios locais; e 6) através de catálogos telefônicos da região para identificar locais de trabalho em potencial.

Após a identificação dos locais de trabalho, um telefonema inicial foi realizado para reunir informações sobre o local de trabalho e investigar potencial interesse em participar do projeto. Para todos os locais de trabalho que atenderam os requisitos iniciais de elegibilidade foi realizada uma tentativa de marcar uma reunião entre os investigadores e os tomadores de decisões (lideres empresariais) chaves para apresentar o projeto e seus requisitos. Após a apresentação inicial, locais de trabalho interessados nomeavam um "contato local" para trabalhar junto à equipe de pesquisa para conduzir as coletas de BHS iniciais de todos trabalhadores.

Após a coleta da BHS, os locais de trabalho que demonstraram apoio contínuo com base em uma forte taxa de resposta do BHS $(\sim 70,0 \%$ em média $)$ e interesse da gerência foram randomizados para um dos dois programas. A randomização foi estratificada com base no número de funcionários (100-300 e 301-600). Este estudo foi aprovado pelo Comitê de Ética da Virginia Tech. (Protocolo \#07/296).
A Adoção neste trabalho é definida como o número, a taxa de participação dos locais de trabalho que satisfazem os critérios de inclusão e a representatividade destes locais de trabalho em relação à população daqueles que foram elegíveis e contatados. Além disso, as razões de inelegibilidade e para o declínio em participar foram relatados quando disponível. Por sua vez, também foram descritos os tipos de locais de trabalho na amostra final e a comparação das taxas de adoção com base no tipo do local de trabalho. Os percentuais foram calculados e análises de qui-quadrado foram realizadas para determinar as diferenças potenciais nas taxas de adoção por nível de variáveis categóricas dos locais de trabalho.

\section{RESULTADOS}

No geral, 119 locais de trabalho foram identificados. A Figura 1 mostra o diagrama de fluxo de recrutamento local de trabalho e de retenção aos seis e 12 meses. Destes, 46 foram considerados não elegíveis para participar do projeto por número reduzido de funcionários $(\mathrm{n}=19)$, muitos empregados $(n=6)$, funcionários não terem acesso à internet $(n=11)$, e locais de trabalho serem dispersos em múltiplos locais $(n=10)$.

Um total de 73 empresas foram consideradas elegíveis para participar do estudo. Estes incluíram nove locais de trabalho classificados como instalações médicas (12,3\%); 13 como centros de produção e distribuição (17,8\%); 16 como grupos profissionais em publicidade, direito, comunicação de vendas, engenharia, seguro e suporte de tecnologia da informação $(21,9 \%)$; quatro como centrais telefônicas (5,5\%); 14 como faculdades e universidades de pequeno porte $(19,2 \%)$; e 17 como agências governamentais (23,3\%).

Destes, 34 recusaram a participação no estudo. As razões incluíam: não estar interessado $(\mathrm{n}=12)$, período de estudo longo $(n=1)$, não ter recursos suficientes $(n=1)$, competição com programas existentes $(n=6)$, fusão de empresas $(n=1)$, e não seguimento com o planejamento $(n=6)$. 
Figura 1. Diagrama de fluxo de recrutamento e retenção de local de trabalho ao longo de 12 meses de execução do programa. Estado da Virginia, EUA, 2007-2010.

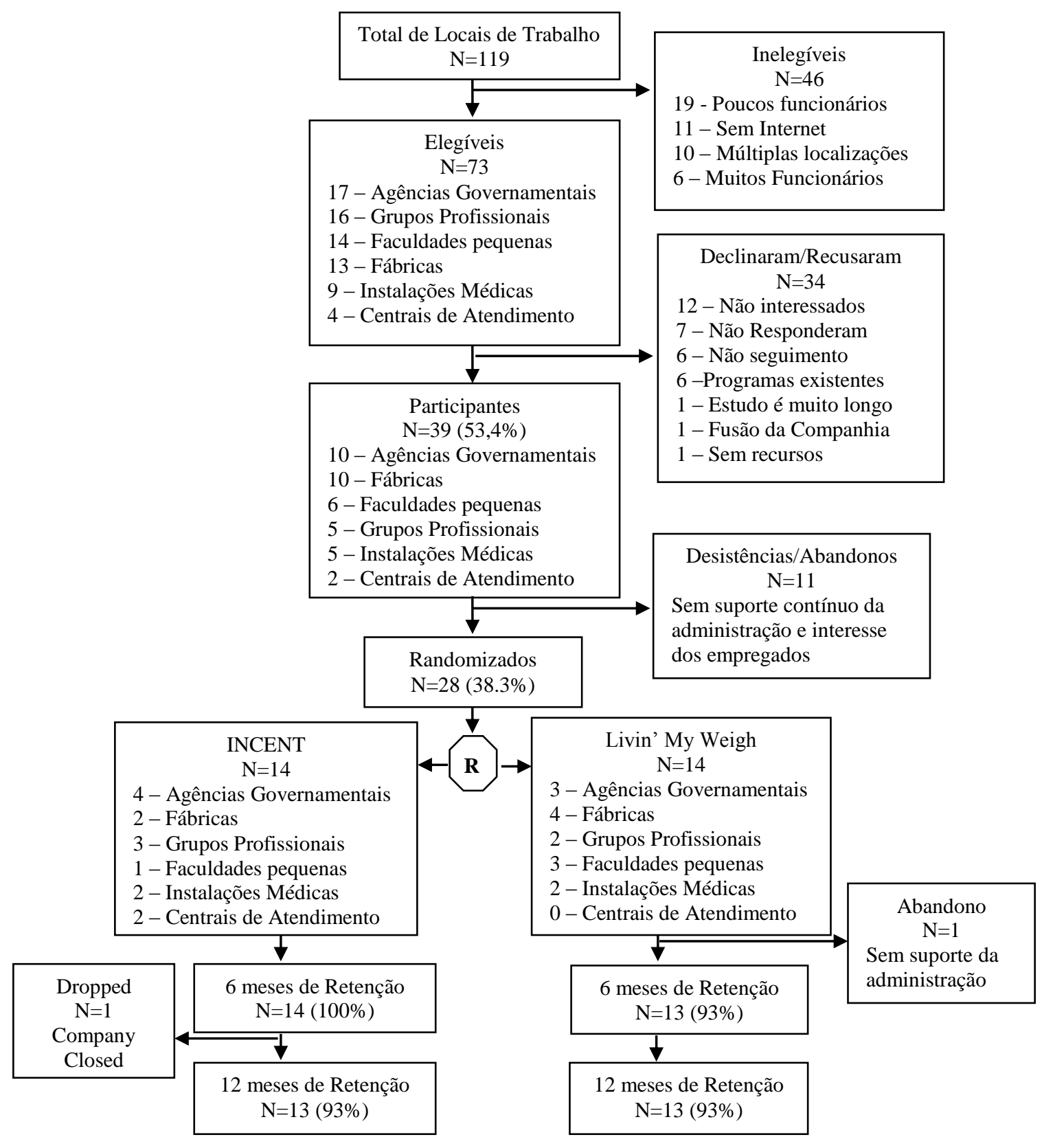

Além disso, sete locais de trabalho não responderam ao convite para participar. Assim, considerou-se 39 locais de trabalho elegíveis $(53,4 \%)$ que concordaram em participar do estudo e realização do inquérito BHS. Destes, 11 descontinuaram / interromperam a participação ao final do BHS devido à falta de apoio contínuo da administração e por baixas taxas de resposta do BHS.
Um total de 28 locais de trabalho (38,3\% índice de adoção) foram randomizados para um dos dois grupos. Esses locais de trabalho incluíam quatro instalações médicas (14,3\%); seis centros de distribuição e produção (21,4\%); cinco grupos profissionais em direito, publicidade, engenharia, vendas e suporte de tecnologia da informação $(17,9 \%)$; dois centros de atendimento $(7,1 \%)$; quatro faculdades e 
universidades de pequeno porte $(14,3 \%)$; e sete agências governamentais $(25,0 \%)$.

Aos seis meses de acompanhamento, uma agência governamental retirou-se do estudo relatando falta de tempo e apoio da administração. Isso reduziu a taxa de adoção para cerca de 37,0\%. Ao fim de 12 meses de acompanhamento um centro de atendimento foi excluído do programa INCENT devido ao fechamento da empresa $(35,6 \%)$.

Ao se comparar as taxas de adoção em vários tipos de local de trabalho não foram observadas diferenças significativas $\left(\chi^{2}(5)=1,688, p=0,890\right)$. No entanto, enquanto a taxa de adoção geral para todos os locais foi de $38,3 \%$, grupos profissionais $(31,0 \%)$ e faculdades de pequeno porte $(28,5 \%)$ tenderam a apresentar taxas mais baixas de adoção. Por outro lado, fábricas $(46,0 \%)$ e agências governamentais $(41,0 \%)$ tenderam a apresentar maiores taxas de adoção. Verificou-se que, em seis e 12 meses, não houve diferenças significativas nas taxas de adoção com base no tipo de programa de perda de peso ou de local de trabalho.

\section{DISCUSSÃO}

No geral, a taxa de aprovação $(53,0 \%)$ foi maior do que outros estudos de promoção da saúde do local de trabalho relataram 15-17. No entanto, uma vez adicionado o requisito de conclusão de $70,0 \%$ do BHS para continuar a participação no estudo, a taxa de adoção final $(38,0 \%)$ ficou alinhada com outros estudos ${ }^{15,16}$.

Essa decisão teve um impacto importante sobre as taxas de adoção e foi feito a fim de garantir que os requisitos da agência financiadora do estudo fossem cumpridos. Estudos futuros baseados no local de trabalho para a promoção da saúde com foco na translação devem diminuir as exigências de participação, tanto quanto possível, a fim de aumentar as potenciais decisões de adoção pelos administradores.

Apesar da variedade de tipos de locais de trabalho aderirem aos programas, não foram observadas diferenças nas taxas de adoção com base no tipo de local de trabalho. No entanto, certos tipos de locais de trabalho mostraram uma tendência de maior recusa do que outros. Adicionalmente, aos 12 meses não houve diferenças com base no grupo que foram designados, o que indica que a randomização e as diferenças de intensidade do programa e as ofertas não levaram os locais de trabalho a interromper a participação.

Os resultados começam a levantar questões importantes sobre decisões de adoção feitas pelos administradores de locais de trabalho, demonstrando que dois programas baseados na internet com intensidades diferentes podem ser aceitáveis para administradores em uma variedade de tipos de local de trabalho.

Além disso, parece que os programas de controle de peso baseados na internet podem ser mais atraentes para administradores de fábricas e agências governamentais. À primeira vista, isso representa um curioso achado dado ao fato de que a maioria das fábricas tem empregados que trabalham longe do computador, enquanto as agências governamentais (ou seja, os departamentos de polícia, delegacias, serviços sociais, companhia de água, Gestão Municipal) incluídas no estudo também tinham profissionais que estavam distantes ou tinham acesso limitado ao computador e internet.

Por outro lado, os administradores de locais onde os funcionários presumidamente teriam acesso mais fácil ao computador e à internet (como grupos profissionais $\mathrm{e}$ faculdades pequenas) eram mais propensos a recusar a participação. Este achado pode ser devido a uma relutância por parte dos empregadores para apoiar programas que incentivam o uso da internet para fins pessoais durante a jornada de trabalho ou porque os empregadores em cargos de decisão podem perceber que os funcionários que usam computadores regularmente no trabalho não têm interesse em acesso a programas que exigem mais tempo de "tela" além do trabalho. 
Estes resultados apresentam considerações importantes para futuros programas quando se leva em conta quais tipos de locais de trabalho devem abordar, para possível inclusão em programas baseados na internet. É evidente que diferentes abordagens e intensidades parecem produzir resultados diferentes em relação à perda de peso ${ }^{6,7,9,18}$.

Acrescenta-se que, enquanto programas baseados na internet têm sido sugeridos como uma possibilidade de melhorar o alcance das intervenções na população alvo ${ }^{19,20}$ os resultados do estudo aqui apresentados sugerem que isso pode não ser verdade para cada configuração de local de trabalho. $\mathrm{Na}$ verdade, se os administradores de certos tipos de locais de trabalho (grupos profissionais e faculdades pequenas) são mais propensos a recusar a participação em programas baseados em internet, isto poderia potencialmente diminuir o alcance dessas intervenções.

Pesquisas futuras devem investigar mais sobre o alcance e a adoção, incluindo a adição de perguntas para melhor entender as crenças e motivações dos administradores que atuam como "porteiros" (elementos chave) para os funcionários sobre a participação nos estudos.

De fato, organizações com gestores que têm um estilo de gestão mais democrático são mais propensos a planejar, adotar e/ou implementar programas de promoção da saúde no local de trabalho do que as organizações com estilos de gestão autoritários $^{21}$. Essa constatação, entretanto, não foi testada e/ou replicada em locais de trabalho que participam em programas de perda de peso baseados na internet.

\section{CONCLUSÃO}

Apesar das taxas de adoção entre certos tipos de organizações (grupos profissionais e faculdades pequenas) terem sido menores na amostra, não se pode ter certeza do porque elas foram menores, podendo haver várias razões.
É necessário reconhecer que as decisões de adoção são normalmente feitas por um indivíduo em uma organização e, assim, para maximizar o alcance (e impacto) é importante trabalhar de maneira a aumentar a adoção, fornecendo mais informações que irão responder às preocupações potenciais desses indivíduos.

Os resultados sugerem ainda que diferentes tipos e quantidades de informação podem ser necessários para os administradores em diferentes locais de trabalho / empresas a serem recrutados para programas baseados em internet.

Finalizando, o conhecimento sobre o alcance, a efetividade, o custo e os requisitos de diferentes tipos de intervenções pode ajudar a fornecer informações mais detalhadas para os administradores nos principais cargos de decisão e pode, potencialmente, aumentar a adoção de programas baseados na internet para locais de trabalho.

\section{REFERÊNCIAS}

1. Flegal KM, Carroll MD, Ogden CL, Curtin LR. Prevalence and trends in obesity among US adults, 1999-2008. JAMA. 2010; 303(3):235-41.

2. Ministério da Saúde (Br). Excesso de peso cresce nos últimos cinco anos [Internet]. Brasília: Ministério da Saúde; [citado em 31/08/11] from: http://portalsaude.saude.gov.br/portalsaude/index.c $\underline{\mathrm{fm} / \text { ?portal=pagina.visualizarNoticia\&codConteudo }=8}$ 73\&codModuloArea $=162 \&$ chamada=excesso-depeso-cresce-nos-ultimos-cinco-anos

3. Terborg JR, Glasgow RE. Worksite interventions. In: Baum A, Newman S, Weinman J, West R, McManus C, orgs. Cambridge handbook of psychology, health, and medicine. Cambridge: Cambridge University Press; 1997. p. 264-8.

4. Bull SS, Gillette C, Glasgow RE, Estabrooks P. Worksite health promotion research: to what extent can we generalize the results and what is needed to translate research to practice? Health Educ Behav. 2003; 30(5):537-49.

5. Estabrooks PA, Glasgow RE. Worksite interventions. In: Baum A, Newman S, Weinman J, West R, McManus C, editores. Cambridge handbook of psychology, health and medicine. $2^{\text {th }}$ ed. Cambridge UK: Cambridge University Press; 2005. p. 264-8.

6. Hennrikus D, Jeffery RW. Worksite intervention for weight control: a review of the literature. Am J Health Promot. 1996; 10(6):471-98. 
7. Jeffery RW, Drewnowski A, Epstein LH, Stunkard AJ, Wilson GT, Wing RR, et al. Long-term maintenance of weight loss: current status. Health Psychol. 2000; 19(1 Suppl):5-16.

8. Wing RR. Behavioral interventions for obesity: recognizing our progress and future challenges. Obes Res. 2003;11(Supplement):3S-6S.

9. Anderson LM, Quinn TA, Glanz K, Ramirez G, Kahwati LC, Johnson DB, et al. Task Force on community preventive services. the effectiveness of worksite nutrition and physical activity interventions for controlling employee overweight and obesity: a systematic review. Am J Prev Med. 2009; 3(4):340-57. 10. Block G, Sternfeld B, Block CH, Block TJ, Norris J, Hopkins D, et al. Development of alive! (a lifestyle intervention via email), and its effect on healthrelated quality of life, presenteeism, and other behavioral outcomes: randomized controlled trial. J Med Internet Res. 2008; 10 (4):e43.

11. Ferdowsian HR, Barnard ND, Hoover VJ, Katcher HI, Levin SM, Green AA, et al. A Multicomponent intervention reduces body weight and cardiovascular risk at a GEICO corporate site. Am J Health Promot. 2010; 24(6):384-7.

12. Akers J, Estabrooks PA, Davy BM. Translational research: bridging the gap between long-term weight loss maintenance research and practice. J Am Diet Assoc. 2010; 110(10):1511-22.

13. Glasgow RE, Vogt TM, Boles SM. Evaluating the public health impact of health promotion interventions: the RE-AIM framework. Am J Public Health. 1999; 89(9):1322-7.

14. You W, Almeida FA, Zoellner JM, Hill JL, Pinard CA, Allen KC, et al. Who participates in internet-based worksite weight loss programs? BMC Public Health. 2011; 11:709. doi:10.1186/1471-2458-11-709

15. Linnan LA, Tate DF, Harrington CB, Brooks-Russell A, Finkelstein E, Bangdiwala S, et al. Organizationaland employee-level recruitment into a worksitebased weight loss study. Clin Trials. 2012; 9(2):21525. doi: 10.1177/1740774511432554.

16. Barbeau EM, Wallace L, Lederman R, Lightman N, Stoddard A, Sorensen G. Recruiting small manufacturing worksites that employ multiethnic, low-wage workforces into a cancer prevention research trial [Internet]. Prev Chronic Dis. 2004 [citado em 22 fev 2014]; 1(3). Disponível em: http://www.ncbi.nlm.nih.gov/pmc/articles/PMC125 3469/pdf/PCD13A04.pdf.

17. Jorgensen MB, Rasmussen CD, Ekner D, Søgaard K. Successful reach and adoption of a workplace health promotion RCT targeting a group of high-risk workers [Internet]. BMC Med Res Methodol. 2010 [citado em 22 fev 2014]; 10:56. Disponível em: http://www.biomedcentral.com/1471-2288/10/56. doi:10.1186/1471-2288-10-56

18. Benedict MA, Arterburn D. Worksite-based weight loss programs: a systematic review of recent literature. Am J Health Promot. 2008; 22(6):408-16.
19. Tate DF, Jackvony EH, Wing RR. Effects of internet behavioral counseling on weight loss in adults at risk for type 2 diabetes. JAMA. 2003; 289(14):1833-6.

20. Petersen R, Sill S, Lu C, Young J, Edington DW. Effectiveness of employee internet-based weight management program. J Occup Environ Med. 2008; 50(2):163-71.

21. Witte K. Managerial style and health promotion programs. Soc Sci Med. 1993; 36(3):227-35.

\section{CONTRIBUIÇÕES}

Fabio Araujo Almeida foi responsável pela condução do estudo, contribuiu no desenho, na coleta e análise de dados, na interpretação e discussão dos resultados, e redação do artigo;

Sarah Stacy Wall conduziu a coleta de dados, contribuiu na interpretação e discussão dos resultados e redação do artigo; Russell Eugene Glasgow contribuiu no desenho, na interpretação e discussão dos resultados, e redação do artigo;

Laura Ann Linnan contribuiu no desenho, na interpretação e discussão dos resultados, e redação do artigo;

Brenda Marie Davy contribuiu no desenho, na interpretação e discussão dos resultados, e redação do artigo;

Jennie Linn Hill contribuiu no desenho, na interpretação e discussão dos resultados, e redação do artigo;

Wendy You conduziu a análise de dados, e contribuiu na interpretação e discussão dos resultados, e redação do artigo;

Paul Andrew Estabrooks contribuiu no desenho, na análise de dados, na interpretação e discussão dos resultados, e redação do artigo.

AGRADECIMENTOS

Este trabalho teve apoio do National Institute on Diabetes and Digestive and Kidney Diseases (Grant R01DK 071664-04 and R01DK 071664-04S1) (Estabrooks, PI). 0 conteúdo aqui apresentado é de única responsabilidade dos autores e não representam necessariamente opiniões oficiais do National Institute of Diabetes and Digestive and Kidney Diseases ou do National Institute of Health. 